\title{
Comparación entre traqueostomía percutánea y quirúrgica, experiencia en un centro hospitalario de la Ciudad de México
}

\section{Comparison between percutaneous and surgical tracheostomy, a single-center experience in Mexico City}

\author{
Alan de Jesús Martínez-Salas, ${ }^{*}$ Patricio Santillán-Doherty, \\ Héctor Aguirre-Mariscal," Rosa Emilia Rivera-Saldana, ${ }^{\S}$ Francisco Becerra-Aguilar, ${ }^{\S}$ \\ Vania Cázares-García," Ariel de Jesús Martínez-Oñate ${ }^{\S}$
}

\footnotetext{
*Hospital General «Dr. Manuel Gea González»; ${ }^{\ddagger}$ Instituto Nacional de Enfermedades Respiratorias Ismael Cosío Villegas; ${ }^{\S}$ Hospital Ángeles Pedregal, Ciudad de México; "Hospital de Pediatría, Centro Médico Nacional, Siglo XXI;

"Hospital General de México «Dr. Eduardo Liceaga».
}

\begin{abstract}
RESUMEN. Introducción: La traqueostomía por técnica percutánea es una técnica de fácil realización, mínimas complicaciones y el estándar de oro en la mayoría de los países; sin embargo, en México aún no se utiliza de una manera amplia. Objetivos: Comparar la técnica percutánea y convencional de traqueostomía en un hospital general de la Ciudad de México. Material y métodos: Estudio retrospectivo mediante la revisión de expedientes clínicos de pacientes adultos, de ambos sexos, sometidos a traqueostomía por técnica percutánea y convencional entre los años 1998 y 2014 en un solo centro hospitalario. Se compararon ambas técnicas en cuanto a tiempo de procedimiento, desenlace quirúrgico, complicaciones, ventilación mecánica y hospitalización. Resultados: Se incluyeron 140 pacientes, 94 con técnica percutánea y 46 convencional, de los cuales 56.4 y $58.7 \%$, respectivamente, el promedio de edad fue de $64 \pm 17$ (media \pm error estándar de la media; $M \pm E E M)$ y $59 \pm 17$ años, respectivamente $(p=0.077)$; el tiempo promedio de procedimiento fue menor con la técnica de traqueostomía percutánea (TPC), $28 \pm 5(\mathrm{M} \pm \mathrm{DE})$ contra $48 \pm 12$ minutos, respectivamente; de las complicaciones presentadas $19.6 \%$ fueron en la técnica convencional (TC) contra $17 \%$ en la percutánea (TPC) $(p=0.815)$. La media de días de estancia hospitalaria fue $31 \pm 5(\mathrm{M} \pm$ EEM) del grupo TC contra $39 \pm 5$ del grupo TPC $(p=0.038)$; la estancia en unidad de cuidados intensivos ( $\mathrm{UCl}$ ) posterior a traqueostomía fue de $13 \pm 3$ del grupo TC contra $16 \pm 5$ del grupo de TPC $(\mathrm{p}=0.038$ ). El porcentaje de altas de $\mathrm{UCl}$ en los primeros 30 días posterior a la traqueostomía fue de $94.7 \%$ en el grupo TPC contra $82.6 \%$ en el grupo TC $(p=0.030)$. Conclusiones: La TPC presenta complicaciones similares a las de la TC; sin embargo, es una técnica que se puede realizar en la cama del paciente en la $\mathrm{UCl}$ sin necesidad de uso de quirófano, de forma segura y rápida. Encontramos diferencias entre ambas técnicas en favor de la TPC en términos de estancia hospitalaria y alta de $\mathrm{UCl}$ en los primeros 30 días después de la traqueostomía; no obstante, no podemos descartar que esto sea atribuible a las patologías de base de cada paciente.
\end{abstract}

Palabras clave: Traqueostomía, traqueostomía percutánea, traqueostomía por dilatación, traqueostomía convencional, ventilación mecánica.
ABSTRACT. Introduction: Percutaneous tracheostomy is considered to be the gold standard technique for performing tracheostomy in most countries, it is an easy to learn technique and has a minimum of complications, however it is not widely used in Mexico. Objectives: To compare the percutaneous (TPC) and conventional tracheostomy (TC) techniques in a general hospital in Mexico. Material and methods: We performed a retrospective review study of the clinical records of all the patients that underwent a tracheostomy procedur between 1998 and 2014 in a single general hospital in Mexico. All tracheostomy procedures, with both precutaneous and conventional techniques, performed in adults, both female and male, were included. Surgical outcome, mechanical ventilation and hospital stay, were compared between both groups. Results: A hundred and forty patients were included, 94 with TPC and 46 with TC, 56.4 vs $58.7 \%$, respectively. Average age was $64 \pm 17$ (age \pm standard error of mean) and $59 \pm 17$ years, respectively $(p=0.077$ ). Average procedure time was inferior with percutaneous technique, $28 \pm 5$ vs $48 \pm 12$ minutes, respectively. Complications with the TPC technique were $19.6 \%$, versus $17 \%$ with the TC group ( $p=0.815)$. Hospital stay in the TPC group was $31 \pm 5$ (mean \pm mean standard error), compared to $39 \pm 5$ days for TC technique $(p=0.038)$. ICU stay after tracheostomy for the TPC group was $16 \pm 5$ and $13 \pm 3$ in the TC technique $(p=0.038)$. ICU discharge within 30 days after tracheostomy was $94.7 \%$ in the TPC group against $82.6 \%$ in the TC group $(p=0.030)$. Conclusions: There is no difference regarding procedural complications between percutaneous tracheostomy technique and conventional tracheostomy, yet, TPC is an accesible, easy to learn and fast technique that holds several advantages; the differences between both techinques in terms of ICU, mechanical ventilation and hospital stay can be attributed to differences related to the different pathologies between both groups.

Keywords: Tracheostomy, percutaneous tracheostomy, dilational tracheostomy, conventional tracheostomy, mechanical ventilation.
Correspondencia: Dr. Ariel de Jesús Martínez Oñate

Hospital General «Dr. Manuel Gea González», Ciudad de México.

Correo electrónico: arielmartinezoñate@gmail.com

Recibido: 15-I-2021; aceptado: 22-III-2021.
Citar como: Martínez-Salas AJ, Santillán-Doherty P, Guzmán-Cedillo AE, Aguirre-Mariscal H, Rivera-Saldana RE, Becerra-Aguilar F, et al. Comparación entre traqueostomía percutánea y quirúrgica, experiencia en un centro hospitalario de la Ciudad de México. Neumol Cir Torax. 2021; 80 (2): 111-117. https://dx.doi.org/10.35366/100992 


\section{INTRODUCCIÓN}

La técnica de traqueostomía percutánea (TPC) fue inicialmente publicada por Ciaglia et al. ${ }^{1}$ en 1985, utilizando dilatadores múltiples, modificada posteriormente para usar un dilatador único. ${ }^{2}$ En 1991 Hazard et al. ${ }^{3}$ publicaron el primer ensayo clínico controlado, comparando ambas técnicas, encontrando superioridad de la TPC. Diversos ensayos clínicos controlados ${ }^{4-6}$ coinciden en mejores resultados con TPC. Dulguerov et al. (1999) 7 publicaron el primer metaanálisis de comparación de las técnicas, concluyendo en la necesidad de realizar más ensayos clínicos controlados. Desde entonces se han publicado diversos ensayos clínicos aleatorizados y metaanálisis para comparar ambas técnicas, ${ }^{8-15}$ mostrando ventajas de la técnica percutánea en cuanto a tiempo de realización y complicaciones. Existe una significativa diferencia en términos de duración del procedimiento en favor de la TPC, tal como lo demuestran las revisiones sistemáticas de Putensen et al..$^{12}$ y Itikhar et al.13 En términos de desenlace y complicaciones únicamente se ha demostrado superioridad respecto a infección de sitio quirúrgico y cicatrización, tal como lo demuestran Putensen et al..$^{12}$ Brass et al..$^{14}$ y Klotz et al. ${ }^{15}$

Desde 1998 en México se han realizado procedimientos de TPC en nuestro centro hospitalario, y aunque ha sido gradualmente adoptada, se usa relativamente poco en los hospitales públicos y privados. Nuestro objetivo es comparar ambas técnicas de traqueostomía y describir la experiencia con la TPC en un hospital general de la Ciudad de México.

\section{MATERIAL Y MÉTODOS}

Realizamos un estudio retrospectivo, descriptivo y comparativo entre las técnicas percutánea y convencional de traqueostomía mediante una revisión de los expedientes del archivo clínico en el Hospital Ángeles Pedregal, Ciudad de México. Se incluyeron pacientes adultos mayores de 18 años, de ambos sexos, desde enero de 1998 hasta octubre de 2014, en quienes se hubiese realizado una traqueostomía. Se excluyeron todos aquellos pacientes cuyo expediente se encontraba incompleto o no estuviera disponible para la obtención de los datos.

La TC comprende la técnica ya conocida de traqueostomía, esencialmente descrita por Jackson en 1909,? realizada en quirófano bajo anestesia general e intubación orotraqueal.

La TPC comprende los principios originalmente descritos por Ciaglia en $1985^{1}$ en un inicio con dilatadores traqueales múltiples y posteriormente con un dilatador traqueal único, ${ }^{2}$ bajo anestesia general e intubación orotraqueal.

Para determinar y comparar el desenlace y la evolución posterior a la traqueostomía se estudiaron los días de es- tancia hospitalaria total, los días de estancia en la unidad de cuidados intensivos $(\mathrm{UCI})$ (posterior a traqueostomía y totales) así como los días de ventilación mecánica (posterior a traqueostomía y totales). También se analizó el destete o retiro de ventilación mecánica y el alta de $\mathrm{UCl}$ en los primeros 30 días posteriores a la realización de traqueostomía.

Realizamos un listado de complicaciones postquirúrgicas y las dividimos en complicaciones tempranas y tardías. Se consideraron complicaciones tempranas: extubación transtraqueostomía, hemorragia o sangrado posoperatorio que requiriera reintervención, colocación no intratraqueal de cánula, laceración de la mucosa de la pars membranosa traqueal, laceración o perforación del esófago, imposibilidad para realizar dilatación o completar procedimiento, muerte durante el procedimiento de traqueostomía o inmediata posterior, pero relacionada con procedimiento, ruptura del globo, recolocación de la cánula por decanulación, cambio prematuro de la cánula (antes de ocho días), retiro de la cánula con reintubación, e infección de herida quirúrgica.

Las complicaciones consideradas como tardías fueron: estenosis subglótica y/o traqueal, persistencia de fístula traqueocutánea después de la decanulación (más de dos semanas), y fístula traqueoesofágica.

Para ambas técnicas se buscó el diagnóstico de ingreso hospitalario, situando los diferentes diagnósticos individuales por grupos para facilitar su análisis como se ha hecho previamente en la literatura., ${ }^{9,16-18}$ Los grupos diagnósticos establecidos fueron: cardiorrespiratorio, neurológico, infectológico, gastroenterológico, oncológico, otro distinto al trauma y trauma.

Dentro de los diagnósticos cardiorrespiratorios se incluyeron enfermedades pulmonares como enfermedad pulmonar obstructiva crónica (EPOC), fibrosis pulmonar, neumonitis, e insuficiencia respiratoria aguda, entre otras, así como enfermedades cardíacas y procedimientos cardiovasculares tales como insuficiencia cardíaca, pericarditis, angioplastías y cirugía cardiovascular, entre otros. Para los diagnósticos neurológicos se incluyó principalmente enfermedad vascular cerebral; el traumatismo craneoencefálico entró en el grupo de trauma. Los diagnósticos infecciosos comprendieron principalmente neumonía y sepsis. Los diagnósticos gastroenterológicos incluyeron pancreatitis aguda complicada, isquemia mesentérica y cirugía abdominal complicada. Dentro del grupo de otros diagnósticos se incluyeron reacciones alérgicas graves y choque anafiláctico.

En estudios previos se han reportado ciertos parámetros que se consideran factores de riesgo de complicaciones transoperatorias y posoperatorias de traqueostomía, ${ }^{19-22}$ por lo que también se buscaron dichos parámetros tales como anticoagulación con heparina, presión positiva al final de espiración (PEEP) $>10 \mathrm{cmH}_{2} \mathrm{O}$, fracción inspirada de oxígeno $\left(\mathrm{FiO}_{2}\right)>50 \%$, infección de tejidos blandos en cuello o evidencia radiológica de lesión cervical. Sin embargo, sólo se pudo registrar la anticoagulación debido 
Neumol Cir Torax. 2021; 80 (2): 111-117

a la falta de datos de los demás parámetros en la mayoría de los expedientes.

Por último, se recolectó información relacionada a la toma de cultivo de secreción bronquial mediante broncofibroscoscopia en los primeros casos, y videobroncofibroscopia a partir de 2006, durante la realización de la TPC, con el fin de determinar si además del beneficio que brinda esta técnica, la toma de cultivo por endoscopia ofrece alguna ventaja al paciente. Todos los procedimientos de TPC se hicieron bajo control endoscópico.

Para variables cuantitativas se usó media más error estándar de la media (EEM) como medida de dispersión. Las variables cualitativas se reportaron en frecuencias y porcentajes. Para las variables cualitativas se utilizó la prueba de $\chi^{2}$ de Pearson y la prueba exacta de Fisher como estadístico analítico; para las variables cuantitativas se utilizó la prueba de U de Mann Whitney para dos grupos y Kruskal Wallis para más de dos grupos con muestras independientes. Se usó el software SPSS para Mac, versión 20.0.

\section{RESULTADOS}

Se registró un total de 164 traqueostomías (percutáneas y quirúrgicas) desde enero de 1998 hasta octubre de 2014.

Se incluyó un total de 140 pacientes que presentaban datos completos, 94 sometidos a la técnica percutánea y 46 a la convencional. Para la técnica percutánea $56.4 \%$ de los sujetos eran hombres y para la convencional $58.7 \%$. La media de edad en el grupo de técnica percutánea fue de $64 \pm 17$ (media \pm EEM) años y de $59 \pm 17$ en la convencional, estadísticamente mayor para el grupo de la técnica percutánea $(p=0.048)$. Las TPC fueron realizadas por

Tabla 1: Diagnósticos de ingreso a unidad de cuidados intensivos de los pacientes sometidos a traqueostomía percutánea y traqueostomía convencional.

\begin{tabular}{|l|c|c|}
\hline $\begin{array}{l}\text { Técnica de } \\
\text { traqueostomía }\end{array}$ & $\begin{array}{c}\text { Técnica percutánea, } \\
\mathrm{N}=94 \\
\mathrm{n}(\%)\end{array}$ & $\begin{array}{c}\text { Técnica convencional, } \\
\mathrm{N}=46 \\
\mathrm{n}(\%)\end{array}$ \\
\hline Grupo diagnóstico & & \\
\hline Cardiorrespiratorio & $27(28.7)$ & $9(19.6)$ \\
\hline Infectológico & $24(25.4)$ & $4(8.7)$ \\
\hline Neurológico & $19(20.2)$ & $5(10.9)$ \\
\hline Trauma & $13(13.8)$ & $7(15.2)$ \\
\hline Oncológico & $4(4.3)$ & $13(28.3)$ \\
\hline $\begin{array}{l}\text { Otro distinto } \\
\text { al trauma }\end{array}$ & $4(4.3)$ & $1(2.2)$ \\
\hline Gastroenterológico & $3(3.2)$ & $7(15.2)$ \\
\hline
\end{tabular}

$p \leq 0.001$, prueba $\chi^{2}$ de independencia. dos distintos cirujanos experimentados del hospital, las TC por cinco distintos cirujanos (incluidos los tres previamente mencionados). En todos los casos la indicación de traqueostomía fue intubación prolongada.

El grupo diagnóstico que se observó con más frecuencia en el total de la población fue el cardiorrespiratorio, 25.7\%, seguido del infectológico en $20.0 \%$. Los diagnósticos se muestran en la Tabla 1; en el grupo TPC predominaron el cardiorrespiratorio y el infectológico (28.7 y 25.4), mientras que en el grupo TC fueron el oncológico y el cardiorrespiratorio (28.3 y $19.6 \%$, respectivamente). Hubo diferencia significativa en los diagnósticos entre ambos grupos, TPC con predominio de infectológicos y neurológicos, y TC con predominio de diagnósticos oncológicos. No hubo diferencia en los diagnósticos de trauma entre las técnicas (Tabla 1).

Los días de estancia hospitalaria fueron $31 \pm 5$ del grupo TC contra $39 \pm$ cinco del grupo TPC, mientras que la estancia en $\mathrm{UCI}$ posterior a traqueostomía fue de $13 \pm$ tres del grupo TC contra $16 \pm$ cinco del grupo de TPC. El grupo de TPC presentó mayor porcentaje de altas de $\mathrm{UCI}$ en los primeros 30 días posteriores a la traqueostomía, 94.7 contra $82.6 \%$ del grupo TC (Tabla 2).

Tras la revisión de expedientes y con los datos encontrados, se analizaron las características en días de estancia hospitalaria, estancia en $\mathrm{UCl}$ y ventilación mecánica entre los distintos grupos diagnósticos, sin tomar en cuenta el grupo de traqueostomía. Se observó diferencia estadísticamente significativa de los días de estancia hospitalaria, estancia en $\mathrm{UCl}$ (posterior a traqueostomía y total), y ventilación mecánica (posterior y total) entre los distintos grupos diagnósticos. El grupo de diagnóstico gastroenterológico mostró el mayor promedio de días de estancia hospitalaria, (51 \pm 12 , media \pm EEM), estancia en $U \mathrm{Cl}$ posterior y total ( $20 \pm$ siete y $36 \pm$ siete, respectivamente) y días de ventilación mecánica total (33 \pm siete). El grupo de diagnóstico cardiorrespiratorio presentó el mayor promedio de días de ventilación mecánica posterior a traqueostomía (20 \pm dos) (Tabla 3).

Se encontró registro del tiempo quirúrgico en 25 (17.9\%) expedientes; 12 de TPC y 13 de TC. La media de tiempo quirúrgico para el grupo de TPC fue de $28 \pm$ cinco minutos, contra $48 \pm 12$ minutos para el grupo de TC $(p=0.077)$.

Las complicaciones fueron similares entre ambas técnicas, $19.6 \%$ (nueve sujetos) de la técnica convencional, contra $17 \%$ (16 sujetos) de la técnica percutánea $(p=0.815)$. En el grupo de TC dos pacientes presentaron dos o más complicaciones simultáneamente. Para el grupo TPC 93.8\% de las complicaciones fueron tempranas y $6.2 \%$ tardías. Para el grupo TC 100\% fueron tempranas (Tabla 4). La complicación que se detectó con más frecuencia en el total de la población fue sangrado posoperatorio, en 4.3\% para ambas técnicas; no se encontró diferencia en la presentación de esta complicación entre los pacientes que recibieron anticoagulación con enoxaparina, 12 horas pre- 
Tabla 2: Estancia hospitalaria, en unidad de cuidados intensivos y ventilación mecánica en pacientes sometidos a traqueostomía percutánea y traqueostomía convencional.

\begin{tabular}{|c|c|c|c|}
\hline Técnica de traqueostomía & $\begin{array}{l}\text { Técnica percutánea, } \\
\qquad(\mathrm{N}=94)\end{array}$ & $\begin{array}{l}\text { Técnica convencional, } \\
\qquad(\mathrm{N}=46)\end{array}$ & $\mathrm{p}$ \\
\hline Estancia hospitalaria total (días; media \pm EEM) & $39 \pm 5^{*}$ & $31 \pm 5$ & 0.038 \\
\hline Estancia UCI posterior a traqueostomía (días; media \pm EEM) & $16 \pm 5$ & $13 \pm 3$ & 0.038 \\
\hline Estancia total en UCl (días; media \pm EEM) & $26 \pm 5$ & $20 \pm 4$ & NS \\
\hline Días de ventilación mecánica posterior a traqueostomía (días; media \pm EEM) & $16 \pm 5$ & $8 \pm 3$ & NS \\
\hline Total de días de ventilación mecánica (días; media \pm EEM) & $25 \pm 5$ & $25 \pm 4$ & NS \\
\hline Alta de $\mathrm{UCl}$ en los primeros 30 días postraqueostomía, $n(\%)$ & $89(94.7)$ & $38(82.6)$ & 0.030 \\
\hline Destete de ventilador en los primeros 30 días postraqueostomía, n (\%) & $88(93.6)$ & $39(84.8)$ & NS \\
\hline
\end{tabular}

*Un paciente del grupo de TPC presentó estancia intrahospitalaria total de 472 días.

$\mathrm{EEM}=$ error estándar de la media, $\mathrm{UCl}=$ unidad de cuidados intensivos, $\mathrm{NS}=$ no significativa.

vias al procedimiento y los que no la recibieron $(\mathrm{p}=1.00)$; 27 sujetos (28.7\%) del grupo TPC y siete sujetos (15.2\%) del grupo de TC $(p=0.95)$ recibieron anticoagulación dentro de las 12 horas previas al procedimiento.

Hubo un total de 24 defunciones intrahospitalarias en la población general, ninguna atribuible a la realización de traqueostomía, 17 (18.1\%) en el grupo TPC y siete (15.2\%) en el grupo TC $(p=0.813)$.

Se hizo lavado bronquioalveolar con cultivo en un total de 63 pacientes, 60 (95.2\%) del grupo TPC y tres (4.8\%) del grupo de TC ( $p<0.001)$ (a pesar de no ser la videobroncofibroscopia parte del procedimiento de la TC), esta diferencia se explica por el acceso inmediato que se tiene a la vía aérea durante la videobroncoscopia que se realiza durante el mismo procedimiento de la TPC. Del total de los lavados 32 (53.3\%) resultaron positivos para microorganismos patógenos en los cultivos, 30 (93.7\%) en el grupo TPC y dos (6.3\%) del grupo TC $(p=0.608)$. Del total de muestras de lavado bronquioalveolar, 60\% (24 muestras) resultaron positivas para bacterias únicamente, $12.5 \%$ (cinco muestras) para hongos, y $27.5 \%$ (11 muestras) para ambos. El microorganismo prevalente en las muestras de lavado bronquioalveolar fue Candida sp. con 46.7\% (14 pacientes) del total de los cultivos positivos. La bacteria más comúnmente aislada fue Pseudomonas aeruginosa en 40\% (12 pacientes) de los cultivos positivos.

\section{DISCUSIÓN}

En nuestra experiencia con la técnica de TPC, observamos que es un procedimiento cuya aceptación en un servicio de $\mathrm{UCl}$ sin experiencia previa con esta técnica toma un tiempo largo; sin embargo, al comprobar sus evidentes ventajas sobre la técnica convencional fue rápidamente aceptada por el personal médico de la $\mathrm{UCl}$ y por los médicos tratantes que tenían a sus pacientes en la misma. Tras la adquisición de experiencia y utilizando una técnica estandarizada obtuvimos resultados con mínima morbimortalidad.

La revisión de expedientes para la comparación de la TPC y TC comprendió un período más largo (ocho años) que el realizado en otros estudios retrospectivos. ${ }^{21,23}$ Debido a que sólo se efectuó en un centro hospitalario, en el cual sólo tres equipos quirúrgicos realizan esta técnica, el número de sujetos incluidos es menor que en otros reportes. En términos demográficos no hubo diferencia entre las técnicas en cuanto al género; la media de edad sí fue mayor en el grupo TPC, lo cual es relevante, ya que según Pandian et al..$^{18}$ la edad es un factor de mal pronóstico en pacientes sometidos a traqueostomía.

Comparada con la TC, la técnica percutánea es más accesible y se puede realizar con mayor velocidad, tal y como se ha demostrado previamente; 9,16,17,24 por lo tanto, en manos experimentadas y un equipo quirúrgico bien integrado es quizá la mejor técnica de traqueostomía.

En el hospital los equipos quirúrgicos que realizan TPC tienen ya varios años de experiencia, dos de ellos iniciaron en 1998 con los primeros casos de TPC, acumulando ya más de 15 años de práctica; es probablemente que por este motivo en nuestro estudio ambas técnicas, TPC y TC presentaron porcentajes de complicaciones muy similares concordando con la literatura. $10,17,24,25$ Es relevante mencionar que Barbetti et al., ${ }^{26}$ habiendo observado una diferencia significativa entre ambas técnicas, reportan un porcentaje de complicaciones para la técnica convencional idéntico al encontrado en nuestro estudio.

La complicación más frecuente en ambos grupos fue hemorragia posoperatoria y aunque no se detectó significancia estadística, lo cual concuerda con estudios previos, ${ }^{10,17}$ el porcentaje de sangrado postraqueostomía fue dos veces mayor con la TC. No se requirió transfusión en ningún caso de los que tuvieron hemorragia posoperatoria con la TPC. 
En estos casos la hemorragia se controló con sutura en forma de jareta alrededor del estoma traqueal o puntos en cruz a los lados de la cánula de traqueostomía, además de aplicación de material de celulosa hemostático en algunos casos. Las maniobras hemostáticas se hicieron en la cama de los pacientes en la UCl.

En muchos estudios se considera la presencia de infección de estoma traqueal como una complicación aparte, nosotros encontramos que aunque no hubo significancia estadística, la técnica percutánea mostró una tasa de infecciones menor que la TC coincidiendo con otros reportes. ${ }^{10,17,27}$ De forma adicional el porcentaje de infección de herida quirúrgica en el grupo TPC fue menor que el descrito en literatura previa. 9,10,20,27

En un metaanálisis, Delaney et al. ${ }^{10}$ concluyeron que no existe diferencia significativa entre las técnicas en cuanto a estancia en $\mathrm{UCl}$ y días de ventilación mecánica; sin embargo, observamos que la técnica percutánea presentó una media de días de estancia hospitalaria total y de estancia en UCl postraqueostomía mayor que el grupo de TC. Oggiano et al. ${ }^{17}$ sí reportan una media de días de estancia en UCI mayor en la TPC en comparación con TC. En nuestro estudio esto pudo deberse a distintos factores, primero, en el grupo TPC hubo un paciente hospitalizado 472 días, de los cuales 422 estuvo en la $\mathrm{UCl}$, mientras que en el grupo de TC el máximo de estancia hospitalaria fue de 130 días en un paciente, y el máximo de estancia en $\mathrm{UCl}$ posterior a la traqueostomía fue de 84 días en otro paciente. Otro factor que probablemente influyó en este resultado es que la mayoría de los pacientes en el grupo TPC se encontraban dentro del grupo de diagnóstico cardiorrespiratorio e infectológico, los cuales presentaron promedios de días de estancia hospitalaria superiores al grupo de diagnóstico oncológico, en éste se encontraron la mayoría de los casos del grupo TC. Ante estos hallazgos, al analizar el porcentaje de pacientes que fueron dados de alta de la $\mathrm{UCl}$ en los primeros 30 días posteriores a la traqueostomía, observamos que fue significativamente mayor en el grupo de TPC, por lo tanto, independientemente del total de días de estancia hospitalaria, la técnica percutánea presenta un mejor desenlace en los primeros 30 días postraqueostomía en comparación con la TC, reflejándose en mayor porcentaje de altas tempranas de la UCl.

Todos los procedimientos de TPC se hicieron bajo control broncofibroscópico al inicio y videobroncofibroscópico desde 2006. La realización de videobroncofibroscopia durante la técnica percutánea ofrece una ventaja diagnóstica y terapéutica sobre la TC.

\section{CONCLUSIONES}

Ambas técnicas son similares en términos de complicaciones, estancia hospitalaria total y en terapia intensiva, pero la técnica percutánea supone ventajas adicionales que son útiles en el contexto de todos los pacientes sometidos a ventilación mecánica, dentro de las cuales destacan: facilidad de realizarse en la cama del paciente, menor tiempo quirúrgico de la técnica (aun sin tomar en cuenta el tiempo de preparación y traslado del paciente), su excelente desenlace en términos de estancia en $\mathrm{UCl}$ (mayor tasa de alta de $\mathrm{UCl}$ en los primeros 30 días postraqueostomía) así como la ventaja adicional que proporciona la videofibro-

Tabla 3: Estancia hospitalaria, en unidad de cuidados intensivos, y ventilación mecánica entre los distintos grupos diagnósticos de pacientes sometidos a alguna técnica de traqueostomía.

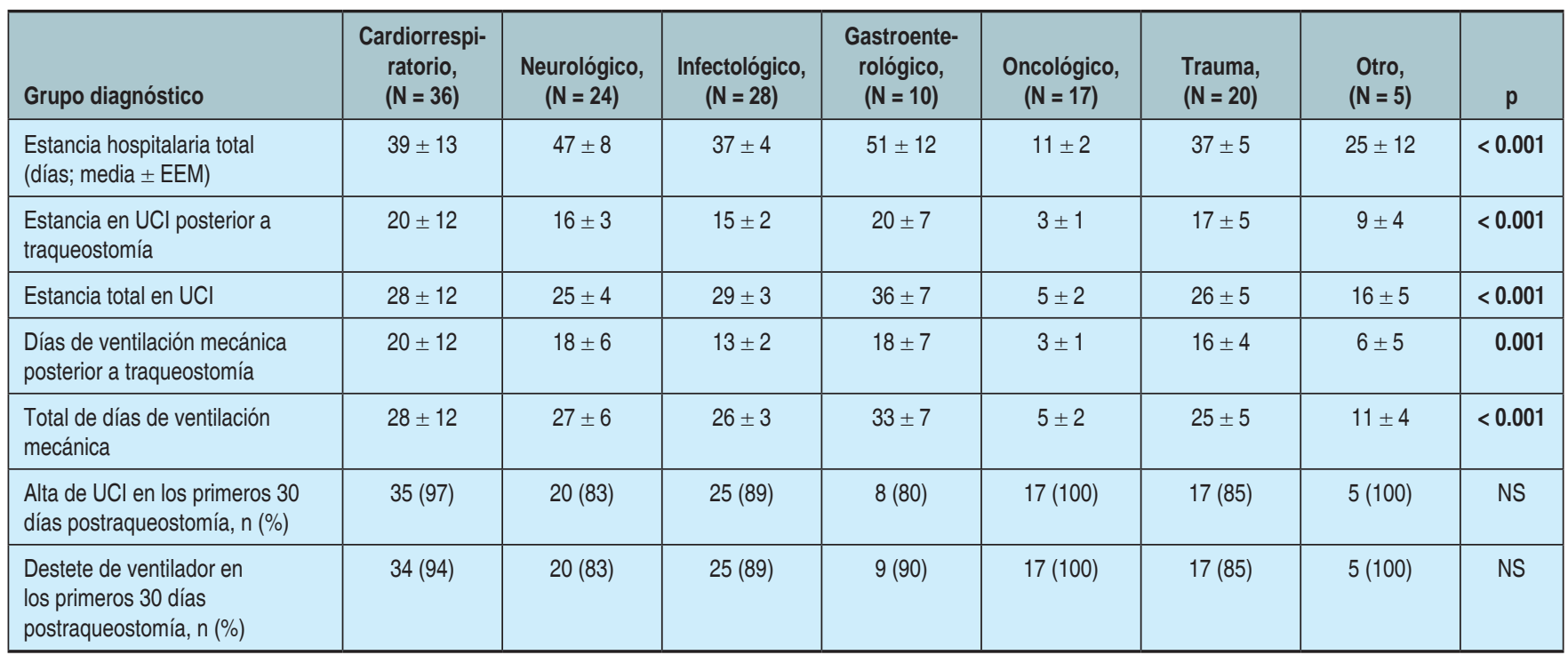

EEM = error estándar de la media, $\mathrm{UCl}=$ unidad de cuidados intensivos, $\mathrm{NS}=$ no significativa. 
Tabla 4: Complicaciones postraqueostomía presentadas en ambas técnicas (traqueostomía percutánea y traqueostomía convencional).

\begin{tabular}{|c|c|c|c|}
\hline Técnica de traqueostomía & $\begin{array}{c}\text { Técnica percutánea, } \\
(\mathrm{N}=94) \\
\mathrm{n}(\%)\end{array}$ & $\begin{array}{l}\text { Técnica convencional, } \\
\qquad(\mathrm{N}=46) \\
\mathrm{n}(\%)\end{array}$ & $\mathrm{p}$ \\
\hline \multicolumn{4}{|l|}{ Complicaciones tempranas } \\
\hline Extubación transtraqueostomía & $2(2.1)$ & 0 & NS \\
\hline Sangrado posoperatorio que requiere intervención & $4(4.3)$ & $4(8.7)$ & NS \\
\hline Laceración de pars membranosa & $2(2.1)$ & 0 & NS \\
\hline Ruptura de globo de cánula & $1(1.1)$ & $2(4.3)$ & NS \\
\hline Recolocación temprana de cánula & $1(1.1)$ & $2(2.2)$ & NS \\
\hline Cambio temprano de cánula & $3(3.2)$ & $1(2.2)$ & NS \\
\hline Retiro temprano de cánula sin recolocación o cambio & $1(1.1)$ & 0 & NS \\
\hline Infección de herida quirúrgica & $1(1.1)$ & $3(6.5)$ & NS \\
\hline \multicolumn{4}{|l|}{ Complicaciones tardías } \\
\hline Estenosis subglótica & $1(1.1)$ & 0 & NS \\
\hline Total de complicaciones & $16(17.0)$ & $12(26.0)^{*}$ & \\
\hline
\end{tabular}

*En el grupo de técnica convencional un sujeto presentó tres complicaciones diferentes; otro tuvo dos complicaciones diferentes. NS = no significativa.

broncoscopia. Todo esto la convierte en la técnica ideal de traqueostomía tanto para cirujanos entrenados en la técnica como para quienes están en entrenamiento quirúrgico.

\section{Agradecimientos}

Dr. Luis Ize Lamache ${ }^{\dagger}$.

A todos los médicos tratantes e intensivistas que han depositado su confianza en nosotros para la realización de los procedimientos en sus pacientes.

\section{REFERENCIAS}

1. Ciaglia P, Firsching R, Syniec C. Elective percutaneous dilatational tracheostomy. A new simple bedside procedure; preliminary report. Chest. 1985;87(6):715-719. Available in: https://doi.org/10.1378/ chest.87.6.715

2. Byhahn C, Lischke V, Scheifler G, Westphal K. [Ciaglia blue rhino: a modified technique for percutaneous dilatation tracheostomy. Technique and early clinical results]. Der Anaesthesist. 2000;49(3):202206. Available in: https://doi.org/10.1007/s001010050815

3. Hazard P, Jones C, Benitone J. Comparative clinical trial of standard operative tracheostomy with percutaneous tracheostomy. Crit Care Med. 1991;19(8):1018-1024. Available in: https://doi. org/10.1097/00003246-199108000-00008

4. Crofts SL, Alzeer A, McGuire GP, Wong DT, Charles D. A comparison of percutaneous and operative tracheostomies in intensive care patients. Can J Anaesth. 1995;42(9):775-779. Available in: https:// doi.org/10.1007/bf03011175

5. Holdgaard HO, Pedersen J, Jensen RH, Outzen KE, Midtgaard T, Johansen LV, et al. Percutaneous dilatational tracheostomy versus conventional surgical tracheostomy. A clinical randomised study. Acta Anaesthesiol Scand. 1998;42(5):545-550. Available in: https:// doi.org/10.1111/j.1399-6576.1998.tb05164.x

6. Muttini S, Melloni G, Gemma M, Casati A, Carretta A, Giudici D, et al. Percutaneous or surgical trachetomy. Prospective, randomized comparison of the incidence of early and late complications. Minerva Anestesiol. 1999;65(7-8):521-527.

7. Dulguerov P, Gysin C, Perneger TV, Chevrolet JC. Percutaneous or surgical tracheostomy: a meta-analysis. Crit Care Med. 1999;27(8):16171625. Available in: https://doi.org/10.1097/00003246-199908000-00041

8. Freeman $\mathrm{BD}$, Isabella K, Cobb JP, Boyle 3rd WA, Schmieg Jr RE, Kolleff $\mathrm{MH}$, et al. A prospective, randomized study comparing percutaneous with surgical tracheostomy in critically ill patients. Crit Care Med. 2001;29(5):926-930. Available in: https://doi. org/10.1097/00003246-200105000-00002

9. Antonelli M, Michetti V, Di Palma A, Conti G, Pennisi MA, Arcangeli $A$, et al. Percutaneous translaryngeal versus surgical tracheostomy: A randomized trial with 1-yr double-blind follow-up. Crit Care Med. 2005:33(5):1015-1020. Available in: https://doi.org/10.1097/01. ccm.0000163401.77581.86

10. Delaney A, Bagshaw SM, Nalos M. Percutaneous dilatational tracheostomy versus surgical tracheostomy in critically ill patients: a systematic review and meta-analysis. Crit Care. 2006;10(2):R55. Available in: https://doi.org/10.1186/cc4887

11. Higgins KM, Punthakee X. Meta-analysis comparison of open versus percutaneous tracheostomy. Laryngoscope. 2007;117(3):447-454. Available in: https://doi.org/10.1097/01.mlg.0000251585.31778.c9

12. Putensen C, Theuerkauf N, Guenther U, Vargas M, Pelosi P. Percutaneous and surgical tracheostomy in critically ill adult patients: a meta-analysis. Crit Care. 2014;18(6):544. Available in: https://doi. org/10.1186/s13054-014-0544-7

13. Itikhar IH, Teng S, Chimmel M, Duran C, Sardi A, Islam S. A network comparative meta-analysis of percutaneous dilational tracheostomies 
using anatomic landmarks, bronchoscopic and ultrasound guidance versus open surgical tracheostomy. Lung. 2019;197(3):267-275. Available in: https://doi.org/10.1007/s00408-019-00230-7

14. Brass P, Hellmich M, Ladra A, Ladra J, Wrzosek A. Percutaneous techniques versus surgical techniques for tracheostomy. Cochrane Database Syst Rev. 2016;7(7):CD008045. Available in: https://doi. org/10.1002/14651858.cd008045.pub2

15. Klotz R, Probst P, Deininger M, Klaiber U, Grummich K, Diener MK, et al. Percutaneous versus surgical strategy for tracheostomy: a systematic review and meta-analysis of perioperative and postoperative complications. Langenbecks Arch Surg. 2018;403(2):137-149. Available in: https://doi.org/10.1007/s00423-017-1648-8

16. Beltrame F, Zussino M, Martinez B, Dibartolomeo S, Saltarini M, Vetrugno L, et al. Percutaneous versus surgical bedside tracheostomy in the intensive care unit: A cohort study. Minerva Anestesiol. 2008;74(10):529-535.

17. Oggiano M, Ewing S, Hecker E. A comparison of percutaneous dilatational tracheostomy versus conventional surgical tracheostomy. Pneumologie. 2014;68(5):322-328. Available in: https://doi. org/10.1055/s-0034-1365198

18. Pandian V, Gilstrap DL, Mirski MA, Haut ER, Haider AH, Efron DT, et al. Predictors of short-term mortality in patients undergoing percutaneous dilatational tracheostomy. J Crit Care. 2012;27(4):420. e9-420.15. Available in: https://doi.org/10.1016/j.jcrc.2011.10.003

19. Kornblith LZ, Burlew CC, Moore EE, Haenel JB, Kashuk JL, Biffl WL, et al. One thousand bedside percutaneous tracheostomies in the surgical intensive care unit: time to change the gold standard. J Am Coll Surg. 2011;212(2):163-170. Available in: https://doi.org/10.1016/j. jamcollsurg.2010.09.024

20. Al-Ansari MA, Hijazi MH. Clinical review: percutaneous dilatational tracheostomy. Crit Care. 2006;10(1):202. Available in: https://doi. org/10.1186/cc3900
21. Madero Pérez J, Vidal Tegedor V, Abizanda Campos R, Cubedo Bort M, Álvaro Sánchez R, Micó Gómez M. Traquesotomía percutánea en pacientes ventilados. Med Intensiva. 2007;31(3):120-125. Available in: https://doi.org/10.1016/s0210-5691(07)74789-7

22. Fikkers BG, Staatsen M, van den Hoogen FJ, van der Hoeven JG. Early and late outcome after single step dilatational tracheostomy versus the guide wire dilating forceps technique: A prospective randomized clinical trial. Intensive Care Med. 2011;37(7):1103-1109. Available in: https://doi.org/10.1007/s00134-011-2222-4

23. Stripf T, Ali M, Mewes T, Mann WJ. [Percutaneous dilatative tracheostomy versus conventional surgical tracheostomy: a retrospective trial]. Laryngorhinootologie. 2003;82(4):281-285. Available in: https://doi.org/10.1055/s-2003-38936

24. Engels PT, Bagshaw SM, Meier M, Brindley PG. Tracheostomy: From insertion to decannulation. Can J Surg. 2009;52(5):427-433.

25. Kettunen WW, Helmer SD, Haan JM. Incidence of overall complications and symptomatic tracheal stenosis is equivalent following open and percutaneous tracheostomy in the trauma patient. Am J Surg. 2014;208(5):770-774. Available in: https://doi.org/10.1016/j. amjsurg.2013.12.036

26. Barbetti JK, Nichol AD, Choate KR, Bailey MJ, Lee GA, Cooper DJ. Prospective observational study of postoperative complications after percutaneous dilatational or surgical tracheostomy in critically ill patients. Crit Care Resusc. 2009;11(4):244-249.

27. Park H, Kent J, Joshi M, Zhu S, Bochicchio GV, Henry S, et al. Percutaneous versus open tracheostomy: comparison of procedures and surgical site infections. Surg Infect (Larchmt). 2013;14(1):21-23. Available in: https://doi.org/10.1089/sur.2011.059

Conflicto de intereses: Los autores declaran no tener conflicto de intereses. 